\title{
Influencia de la minería informal sobre la contaminación del medio ambiente y la vulneración del derecho a la vida en Carabayllo. Período $2011^{1}$
}

Influence of Informal Mining on Environmental Pollution and Violations of the Right to Life in Carabayllo. Period 2011.

\section{Resumen}

El objetivo de la investigación es demostrar que la minería informal contamina el medio ambiente y vulnera el derecho a la calidad de vida en el distrito limeño de Carabayllo. El período de estudio es el año 2011; la investigación parte del estudio de los informes técnicos que existen sobre la zona de impacto, emitidos por organismos oficiales, con el fin de lograr propuestas que ayuden al Estado a formalizar la minería informal en la Región Lima, realizando algunas mejoras y/o cambios al D. S. N 045-2010-PCM.

Palabras clave: minería informal, medio ambiente, contaminación.

\begin{abstract}
The objective of this research is to demonstrate how informal mining pollutes the environment and violates the right to quality of life in the Lima district of Carabayllo, the study period is 2011, the research part of the study reports technicians that exist on the impact area, in order to obtain proposals, which will help the State, and formalize the informal mining in the region of Lima, making some improvements and / or changes to D. S. $\mathrm{N}^{\circ}$ 045-2010-PCM.
\end{abstract}

Key words: mining informal, environment, pollution.

\footnotetext{
Esta tesis puede ser revisada en la biblioteca de la Universidad Alas Peruanas.

Título de abogado por la Universidad Alas Peruanas.
} 


\section{Introducción}

La presente investigación busca frenar los impactos negativos ambientales ocasionados por la minería informal que contaminan el medio ambiente en el distrito limeño de Carabayllo, considerando que la Ciencia del Derecho tiene un compromiso para regular los hechos que ocasionan y contaminan nuestra urbe limeña. Constituyendo los conflictos socioambientales una problemática social actual, la presente investigación busca contribuir para regular y resolver las actividades de los mineros informales, a fin de lograr la formalización en un Estado de Derecho con paz social. Somos conscientes de que la contaminación por mercurio es el principal problema que ocasiona la minería artesanal en la extracción del oro.

Esta actividad no solo contamina el medio ambiente, sino que es también responsable de la trata de personas, una problemática nacional e internacional. Siendo la minería informal una actividad que predomina en yacimientos auríferos de tipo veta y aluvial, tiene apoyo de los Gobiernos regionales, responsables directos de formalizar esta actividad con una prioridad indispensable. Esta investigación busca regular la minería informal para que esta ya no contribuya a la contaminación del medio ambiente y vulnere el derecho a una mejor calidad de vida en el distrito de Carabayllo. Como punto central de la propuesta está modificar el D.S. No 045-2010-PCM, de modo que considere en la formalización de la minería informal a la región Lima.

Reconocemos que la política de libre mercado del Perú está fundamentada en la Constitución Política del Estado de 1993. Cabe resaltar que en este marco constitucional se encuentran las inversiones formales, mientras que la minería informal está en pleno proceso de reforma integral (la actividad informal), que es la que contamina y afecta los recursos naturales y pone en riesgo la vida humana, flora y fauna.

La contaminación ambiental es un problema global que afecta a los países ricos y en mayor magnitud a los países pobres. Este es un problema que requiere el esfuerzo consciente de todos para resolverlo, porque el planeta Tierra es de todos, y depende de todos los esfuerzos, legales, sociales, ambientales etc., cuidarlos para tener una vida digna, en especial los pobladores de la zona de estudio.

Esta investigación está direccionada a erradicar la minería informal. A manera de ejemplo, podemos mencionar lo que viene ocurriendo en la región de Madre de Dios, donde la explotación minera informal aurífera está generando una depredación de los recursos naturales (flora, fauna, ríos y suelos) en los territorios de las comunidades nativas, que fueron invadidos por los explotadores informales (los colonos). Esta codicia por la "fiebre de oro" ha influenciado en la alta cotización del oro en US\$ 1800 la onza. Esta cotización hace que sean más vulnerables los derechos humanos, por la forma como se realiza la explotación del valioso 
mineral. Esta actividad ilegal no solo contamina el ambiente, sino que también produce otros delitos conexos, porque a la región en mención se le identifica como un importante lugar de origen y destino de la trata, principalmente con fines de explotación sexual y laboral. A nivel local, Lima Metropolitana se abastece de tres cuencas hidrográficas: Chillón, Rímac y Lurín. Según el Comercio (revista Somos N ${ }^{\circ}$ 1218, 2010), la cuenca del río Chillón presenta un problema de minería aurífera que contamina sus aguas con mercurio. Ahora, Salazar (2009) detalló que Río Seco es un poblado entre Carabayllo y Huarochirí, con unas 300 familias que viven entre los cultivos de lechuga y la extracción ilegal de oro. Al respecto, la Dirección Regional de Minería, a la fecha del estudio, no se decidió a intervenir en la zona informal. De igual forma, como una respuesta a la alarma descrita, el Ministerio de Salud tomó una muestra a treinta vecinos de esta comunidad y se les detectó que tenían concentraciones de mercurio en su organismo, información letal que motiva nuestra investigación.

\section{Objetivo general}

Demostrar que la minería informal contribuye a la contaminación del medio ambiente, vulnerando el derecho a una mejor calidad de vida en el distrito de Carabayllo, a través del análisis de informes y estadísticas públicas, con el fin de proponer al Estado la modificación del D.S. No 045-2010-PCM, para que considere en la formalización de la minería informal a la región Lima. (Período de estudio: año 2011).

\section{Objetivos especificos}

1. Reforzar a través de la literatura que las materias tóxicas contribuyen a la contaminación del suelo, con el fin de proponer regulación y efectividad a los instrumentos de gestión ambiental; para que disminuyan los daños en la fertilidad de la tierra.

2. Constatar que los relaves mineros producen la contaminación del agua en la cuenca del río Chillón, a fin de proponer efectividad al Reglamento Ambiental para las Actividades Mineras - D.S. N 059-93-EM, con el propósito de disminuir la degradación ambiental.

3. Determinar que los vehículos en mal estado que usan combustibles fósiles contribuyen a la contaminación del aire, con el propósito de proponer efectividad a la Ley de Promoción del Uso Eficiente de la Energía -Ley No 27345, para proteger la salud personal.

\section{Hipótesis general}

Si se incrementa la actividad de laminería informal, entonces se contribuiría a la contaminación del medio ambiente, vulnerando el derecho a una mejor calidad de vida en el distrito de Carabayllo durante el año 2011. 


\section{Hipótesis secundarias}

1. A mayor existencia de materias tóxicas, mayor sería la contaminación del suelo.

2. A mayor vertimiento de los relaves mineros, mayor sería la contaminación del agua en la cuenca del río Chillón.

3. A mayores vehículos en mal estado que usan combustibles fósiles, mayor sería la contaminación del aire.

\section{Conceptos importantes}

Ambiente o medio ambiente. Es el conjunto de elementos sociales, económicos, culturales, bióticos y abióticos que interactúan en un espacio y tiempo determinado.

Calidad de vida. Es el cumplimiento por parte del Estado de sus funciones esenciales para satisfacer las necesidades básicas de alimentación, vivienda, salud y educación a través de servicios públicos adecuados que lleguen a los sujetos que no estén en condiciones de proveerse por sí de tales recursos. Es un complemento necesario del medio ambiente.

Concesión minera. Es un acto administrativo emanado de la autoridad competente, que autoriza a realizar una actividad de exploración-desarrollo-explotación o de beneficio, labor general o transporte minero, según sea el caso.

Daño ambiental. Es toda actividad humana individual o colectiva que ataca los elementos del patrimonio ambiental, que causa un daño social por afectar intereses pertenecientes a la comunidad, y no tiene por finalidad la tutela del interés de un sujeto en particular.

Exploración. Es una actividad que conduce "a demostrar las dimensiones, posición, características mineralógicas, reservas y valores de los yacimientos minerales” (Art. 8, primer párrafo, del TUO). Esta actividad requiere concesión minera.

Explotación. "Es la actividad de extracción de los minerales contenidos en un yacimiento" (Art. $8^{\circ}$, tercer párrafo, del TUO). Esta actividad requiere concesión minera.

Formalización. Es el proceso mediante el cual se establecen y administran los requisitos, plazos y procedimientos para que la actividad minera informal pueda cumplir con la legislación vigente.

Gestión ambiental. Es el conjunto de acciones, políticas, regulaciones, principios y la institucionalidad, diseñadas y ejecutadas por el Estado con participación de la sociedad civil, la academia y el sector privado para lograr una ordenación sostenible del ambiente, como presupuesto básico para aspirar al desarrollo sostenible del país y a un ambiente sano. 
Mercurio. Es un elemento metálico líquido, inodoro y de color plata brillante, ligeramente volátil a temperatura ambiente $\left(20^{\circ} \mathrm{C}\right)$. Tanto este como sus derivados son tóxicos, no es biodegradable y forma fácilmente aleaciones con muchos metales, excepto con el hierro.

Minería ilegal. Comprende las actividades mineras que se realizan sin cumplir con las exigencias de las normas de carácter administrativo, técnico, social y ambiental que rigen dichas actividades, y que se ubican en zonas no autorizadas para el ejercicio de dichas actividades, las que son llevadas a cabo por personas naturales o jurídicas o grupos de personas organizadas para tal fin.

Minería informal. Es aquella actividad minera que, teniendo características de minería ilegal, se realiza en zonas autorizadas para la actividad minera. Quienes la realizan han iniciado un proceso de formalización en los plazos y modalidades establecidos en las normas sobre la materia.

\section{Metodología}

\section{Tipo de investigación}

Es de tipo aplicado, porque está fundamentado en las teorías del Derecho Ambiental y Derecho Minero.

\section{Nivel de investigación}

Es descriptiva, porque el objeto es conocer y describir de manera precisa la magnitud del problema socioambiental, identificando las características y las variables que genera. Partimos de una hipótesis de trabajo y luego proponemos las estrategias para formalizar y erradicar la minería informal. Buscamos que la propuesta sea correlacional, al relacionar causa-efecto entre la minería informal y la contaminación del medio ambiente.

\section{Diseño de investigación}

Es propositiva, al determinar la necesidad imperiosa de formalizar la actividad informal para que no siga contaminando el medio ambiente en pro de la naturaleza y de los pobladores del distrito de Carabayllo. Es cuantitativa, porque los contaminantes tienen valores medibles en la estadística; y es cualitativa, porque por la contaminación es expresada en el informe sobre el estado de salud de las personas. Esta investigación es de corte transversal, porque, según el problema principal, es la minería informal la que contribuye a la contaminación del medio ambiente en el distrito de Carabayllo. Esto es parte del análisis de los datos del 2011. 


\section{Métodos de investigación}

Deductivo. Porque la contaminación del medio ambiente en el distrito de Carabayllo debe ser resuelta por el Estado con la formalización de la minería informal.

Inductivo. Porque la minería informal genera distintos problemas de contaminación: contaminación del suelo, contaminación del agua y contaminación del aire.

Analitico-critico. Porque el incremento de la minería informal contribuiría a la contaminación del medio ambiente en el distrito de Carabayllo, y debería ser resuelto por el Estado con la formalización de la minería informal.

Estadístico. Porque según datos estadísticos analizados por la Dirección General de Salud (DIGESA) existe contaminación del medio ambiente en Carabayllo y en Lima Metropolitana.

Muestra

Está conformada por la siguiente Unidad de Análisis:

- Nueve informes que identifican la contaminación del medio ambiente producido por la actividad de la minería informal.

- 01 ingeniero.

\section{Técnicas e instrumentos de recolección de datos}

Técnicas. Constituyen un proceso que involucra investigaciones para recoger respuestas de la realidad. Para este caso se empleó:

- Análisis documental (informes).

Instrumentos. Para la recolección de datos de la realidad se utilizó:

- Guía de análisis documental.

\section{Tipo de estudio}

Validez. Para validar los nueve informes específicos (muestra aleatoria simple), se utilizó la respuesta por análisis dicotómicos de los 24 indicadores.

Confiabilidad. Para determinar la confiabilidad de la Guía de Análisis Documental se utilizó el coeficiente de Kuder-Richardson (KR20).

Para determinar el grado de confiabilidad del instrumento denominado Guía de Análisis Documental, se determinó una muestra de nueve informes con 24 indicadores con análisis de 
respuestas dicotómicas para obtener el Coeficiente de Consistencia Interna KR-20.

$$
r=\left(\frac{k}{k-1}\right)\left[1-\frac{\sum_{i=1}^{k} p_{i}\left(1-p_{i}\right)}{S^{2}}\right]
$$

Donde:

$p_{i}:$ Proporción de indicadores

$k$ : Número de informes

$S^{2}$ : Varianza

Finalmente, según la tabulación de datos de la Guía de Análisis Documental, se obtuvo una confiabilidad de instrumento casi ideal, aplicando el Coeficiente Kuder-Richardson (KR-20), equivalente a 0,973 .

Coeficiente Kuder-Richardson (KR-20): Guía de Análisis Documental:

\begin{tabular}{|c|c|}
\hline & Valor \\
\hline KR-20 & 0.973 \\
\hline
\end{tabular}

Tablas y análisis de datos

Tabla 1. La minería informal

\begin{tabular}{|l|c|c|}
\hline $\begin{array}{l}\text { Indicador P1: Sin título } \\
\text { de concesión minera }\end{array}$ & No $^{\circ}$ de informes & Porcentaje \\
\hline Sí & 5 & $55,55 \%$ \\
\hline No & 4 & $44,44 \%$ \\
\hline Total de informes & 9 & $100 \%$ \\
\hline
\end{tabular}




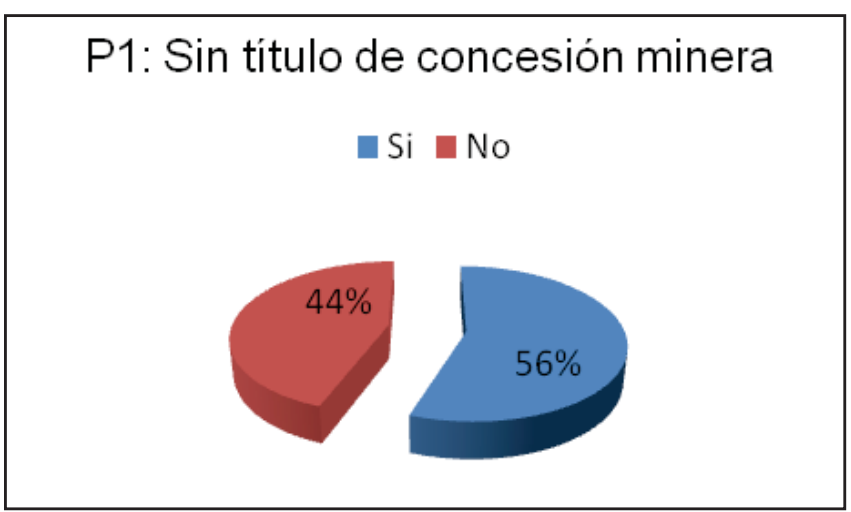

Figura 1

Según el análisis de los nueve informes, respecto a la V.I (minería informal), cuyo indicador es P1 (Sin título de concesión minera), en cinco informes, equivalentes a 56\%, sí se mencionó dicho indicador, y en cuatro informes, equivalentes a $44 \%$, no se mencionó.

Tabla 2. La minería informal

\begin{tabular}{|l|c|c|}
\hline $\begin{array}{l}\text { Indicador P2: Sin contrato } \\
\text { de explotación minera }\end{array}$ & No de informes & Porcentaje \\
\hline Sí & 5 & $55,55 \%$ \\
\hline No & 4 & $44,44 \%$ \\
\hline Total de informes & 9 & $100 \%$ \\
\hline
\end{tabular}

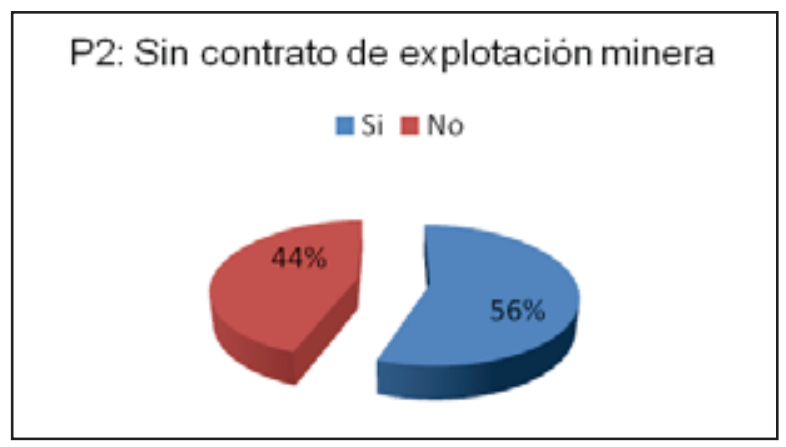

Figura 2

Según el análisis de los nueve informes, respecto a la V.I (minería informal), cuyo indicador es P2 (Sin contrato de explotación minera), en cinco informes, equivalentes a 56\%, sí se mencionó dicho indicador, y en cuatro informes, equivalentes a 44\%, no se mencionó. 
Tabla 3. La minería informal

\begin{tabular}{|l|c|c|}
\hline Indicador P3: Sin permiso legal & $\mathrm{N}^{\circ}$ de informes & Porcentaje \\
\hline Sí & 5 & $55,55 \%$ \\
\hline No & 4 & $44,44 \%$ \\
\hline Total de informes & 9 & $100 \%$ \\
\hline
\end{tabular}

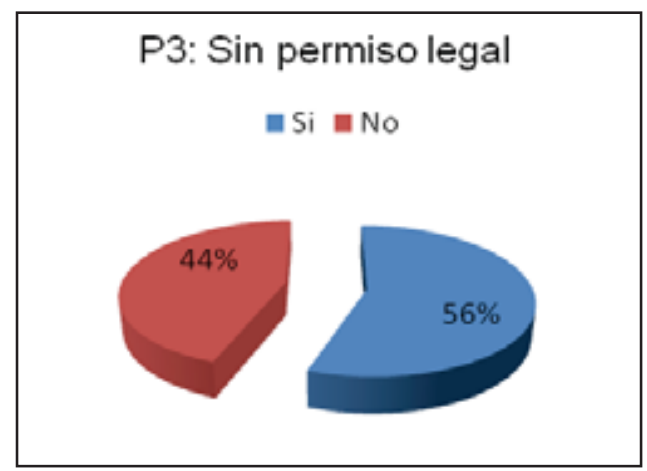

Figura 3

Según el análisis de los nueve informes, respecto a la V.I (minería informal), cuyo indicador es P3 (sin permiso legal), en cinco informes, equivalentes a 56\%, sí se mencionó dicho indicador, y en cuatro informes, equivalentes a $44 \%$, no se mencionó.

\section{Tabla 4. La contaminación del medio ambiente}

\begin{tabular}{|l|r|r|}
\hline $\begin{array}{l}\text { Indicador P4: Introducción } \\
\text { de agentes físicos que superan el } \\
\text { Límite Máximo Permisible (LMP) y y Estándar } \\
\text { de Calidad Ambiental (ECA) }\end{array}$ & No de informes & Porcentaje \\
\hline Sí & 3 & $33,33 \%$ \\
\hline No & 6 & $66,66 \%$ \\
\hline Total de informes & 9 & $100 \%$ \\
\hline
\end{tabular}

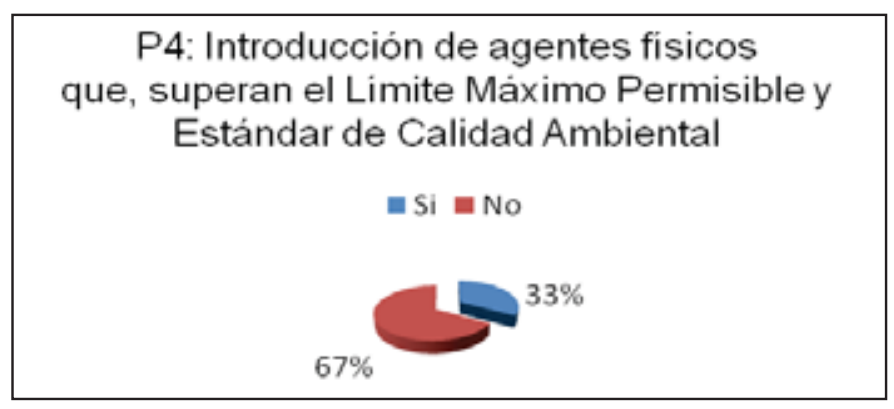

Figura 4 
Según el análisis de los nueve informes, respecto a la V.D (contaminación del medio ambiente), cuyo indicador es P4 (introducción de agentes físicos que superan el Límite Máximo Permisible y Estándar de Calidad Ambiental), en tres informes, equivalentes a 33\%, sí se mencionó dicho indicador, y en seis informes, equivalentes a $67 \%$, no se mencionó.

\section{Tabla 5. La contaminación del medio ambiente}

\begin{tabular}{|l|r|c|}
\hline $\begin{array}{l}\text { Indicador P5: Introducción de agentes químicos } \\
\text { que superan el Límite Máximo Permisible (LMP) } \\
\text { y y Estándar de Calidad Ambiental (ECA) }\end{array}$ & No de informes & Porcentaje \\
\hline Sí & 9 & $100 \%$ \\
\hline No & 0 & $0 \%$ \\
\hline Total de informes & 9 & $100 \%$ \\
\hline
\end{tabular}

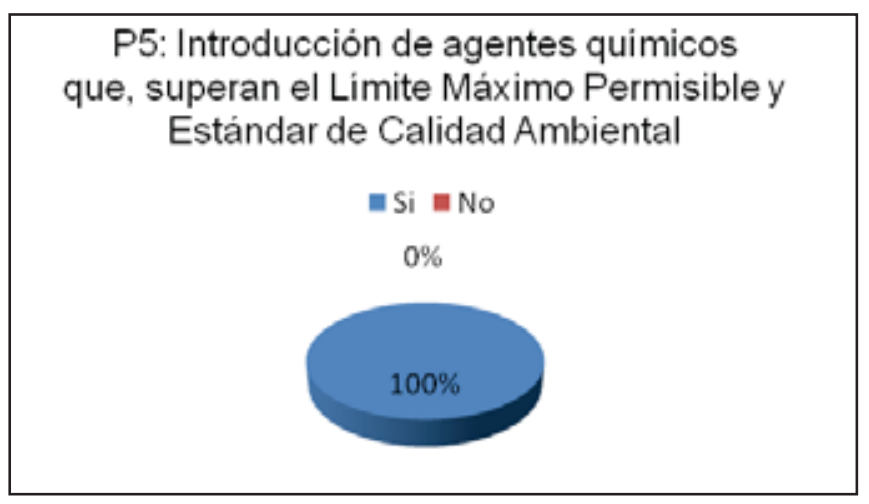

Figura 5

Según el análisis de los nueve informes, respecto a la V.D (contaminación del medio ambiente), cuyo indicador es P5 (introducción de agentes químicos que superan el Límite Máximo Permisible y Estándar de Calidad Ambiental), en nueve informes, equivalentes a $100 \%$, sí se mencionó dicho indicador.

Tabla 6. La contaminación del medio ambiente

\begin{tabular}{|c|c|c|}
\hline $\begin{array}{l}\text { Indicador P6: Introducción de agentes biológicos } \\
\text { que superan el Límite Máximo Permisible (LMP) } \\
\text { y Estándar de Calidad Ambiental (ECA) }\end{array}$ & $\begin{array}{c}\mathrm{N}^{\circ} \text { de } \\
\text { informes }\end{array}$ & Porcentaje \\
\hline Sí & 2 & $22,22 \%$ \\
\hline No & 7 & $77,77 \%$ \\
\hline Total de informes & 9 & $100 \%$ \\
\hline
\end{tabular}




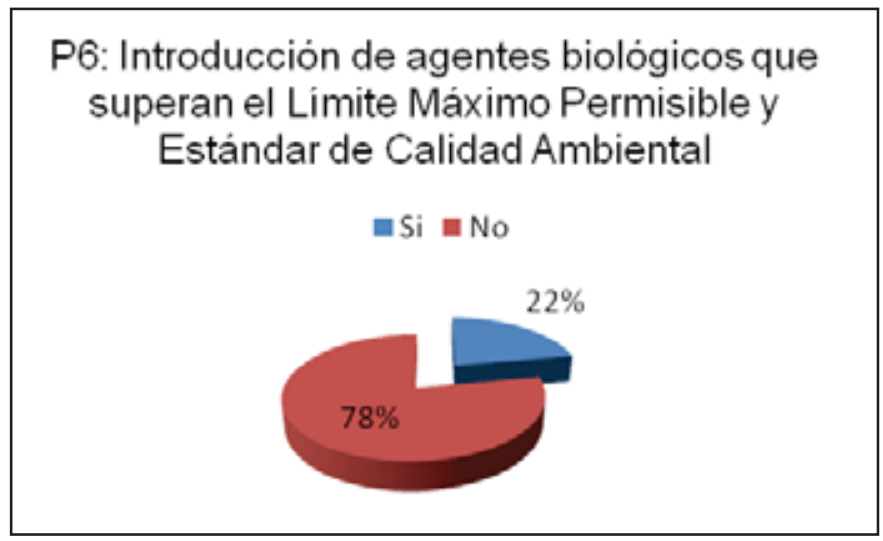

Figura 6

Según el análisis de los nueve informes, respecto a la V.D (contaminación del medio ambiente), cuyo indicador es P6 (introducción de agentes biológicos que superan el Límite Máximo Permisible y Estándar de Calidad Ambiental), en dos informes, equivalentes a 22\%, sí se mencionó dicho indicador, y en siete informes, equivalentes a 78\%, no se mencionó.

Tabla 7. Las materias tóxicas

\begin{tabular}{|c|c|l|}
\hline Indicador P7: Nivel de mercurio & $\begin{array}{l}\text { N}^{\circ} \text { de } \\
\text { informes }\end{array}$ & Porcentaje \\
\hline Sí & 3 & $33,33 \%$ \\
\hline No & 6 & $66,66 \%$ \\
\hline Total de informes & 9 & $100 \%$ \\
\hline
\end{tabular}

P7: Nivel de mercurio

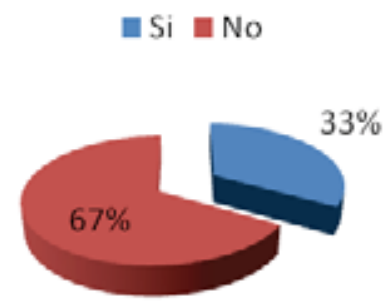

Figura 7 
Según el análisis de los nueve informes, respecto a la V.I (materias tóxicas), cuyo indicador es P7 (nivel de mercurio), en tres informes, equivalentes a 33\%, sí se mencionó dicho indicador, y en seis informes, equivalentes a $67 \%$, no se mencionó dicho indicador.

\section{Conclusiones}

1. El incremento de la actividad en la minería informal contribuye a la contaminación del medio ambiente, hecho que se comprobó por la falta de concesiones mineras y la introducción de agentes físicos, químicos y biológicos que superaron los instrumentos de gestión ambiental, vulnerando el derecho a una mejor calidad de vida en el distrito de Carabayllo durante el año 2011.

2. Debido a una mayor existencia de materias tóxicas, se produce una mayor contaminación del suelo, lo que está demostrado en los informes analizados; principalmente por el nivel de mercurio utilizado para la extracción del oro y el deterioro químico que degrada la calidad del suelo, vulnerando los derechos de los pequeños agricultores del valle.

3. Como consecuencia del mayor vertimiento de relaves mineros, se ocasiona mayor contaminación del agua en la cuenca del río Chillón, hecho que se manifestó en forma relevante en el nivel de afectación a la salud humana por la contaminación química existente, perjudicando a los usuarios del sector.

4. Finalmente, a mayores vehículos en mal estado que usan combustibles fósiles, mayor es la contaminación del aire, lo que se percibe en el nivel de combustión de petróleo, produciendo un alto porcentaje de material particulado, el mismo que ocasiona dificultad en la respiración de la población urbana.

\section{Recomendaciones}

1. Para que la minería informal no contribuya a la contaminación del medio ambiente y vulnere el derecho a una mejor calidad de vida en el distrito de Carabayllo durante el año 2011, el Estado debe modificar el D.S N N $^{\circ}$ 45-2010-PCM para que considere en la formalización de la minería informal a la región Lima.

2. Debido a una mayor presencia de materias tóxicas (principalmente de mercurio, utilizado en la extracción del oro) que incrementan la contaminación del suelo por el deterioro químico, se propone mayor efectividad a los instrumentos de gestión ambiental (LMP y 
ECA), de manera que disminuyan los daños en la fertilidad de la tierra y no se vulneren los derechos de los pequeños agricultores del valle.

3. Como consecuencia de un mayor vertimiento de los relaves mineros, los mismos que producen una mayor contaminación del agua en la cuenca del río Chillón, se propone efectividad al Reglamento Ambiental para las Actividades Mineras - D.S. Nº 059-93-EM, a fin de no perjudicar a los usuarios del sector.

4. Finalmente, para que los vehículos en mal estado que usan combustibles fósiles no contribuyan a la contaminación del aire por la combustión del petróleo, se propone efectividad a la Ley de Promoción del Uso Eficiente de la Energía - Ley N² 27345, para proteger la salud de las personas.

\section{Referencias}

- Andaluz Westreicher, Carlos (2009). Manual de Derecho Ambiental. Perú: Editorial Iustitia.

- Belaúnde Moreyra, Martín (2011). Derecho Minero y Concesión. Perú: Editorial San Marcos E.I.R.L.

- Bustamante Alsina, Jorge. Derecho Ambiental. Buenos Aires, Argentina: Editorial AbeledoPerrot.

- Carhuatocto Sandoval, Henry (2009). Guía del Derecho Ambiental. Perú: Jurista Editores.

- Carruietero Lecca, Francisco (2006). Derecho del Medio Ambiente. Estrategias de Derecho Privado para conservar la naturalezay luchar contra la contaminación ambiental. DeTrazegnies Granda, Fernando. Perú: Jurista Editores, p.193.

- Charpentier, Silvia e Hidalgo, Jessica (1999). Políticas ambientales en el Perú. Agenda Perú.

- Celi Arévalo, Marco (2006). Derecho Ambiental. Hacia un desarrollo sostenible. Volumen I. Perú.

- Chanamé Orbe, Raúl (2009). Comentarios a la Constitución. Perú: Jurista Editores.

- Colegio de Ingenieros del Perú. Primer Simposio Nacional de Medio Ambiente y Seguridad Minera (1997). Roque, Diógenes. Experiencias prácticas en el control de emisiones de mercurio en la pequeña minería aurífera boliviana, p.187. Hruschka, Félix. Control de la contaminación en la minería artesanal y pequeña minería, p.201. 
- Cornejo Ramírez, Enrique (2010). Comercio Internacional. Perú: Editorial San Marcos.

- Hernández Sampieri, Roberto; Fernández-Collado, Carlos; y Baptista Lucio, Pilar (2006). Metodología de la Investigación. México.

- Instituto Peruano de Economía (2011). La tributación minera en el Perú: contribución, carga tributaria y fundamentos conceptuales.

- Lazo Arrasco, Jorge (2011). Pedagogía Universitaria. Perú: Fondo Editorial UAP.

- Ministerio de Energía y Minas (2010). Anuario Minero. Perú.

- Ministerio de Energía y Minas (2011). Guías $N^{\circ} 1$ y $N^{\circ} 2$ para el minero artesanal formal y responsable. Perú.

- Ministerio del Ambiente (2011). Minería aurífera en Madre de Dios y contaminación con mercurio: una bomba de tiempo. Informe preparado por el Instituto de Investigaciones de la Amazonía Peruana - IIAP y el Ministerio del Ambiente. Brack, Antonio; Álvarez, José; e Ipenza, César.

- Novak, Fabián y Namihas, Sandra (2009). La trata de personas con fines de explotación laboral: el caso de la minería aurifera y la tala ilegal de madera en Madre de Dios.Perú: Instituto de Estudios Internacionales (IDEI) de la Pontificia Universidad Católica del Perú y la Organización Internacional para las Migraciones (OIM).

- Naupas Paitán, Humberto; Mejía Mejía, Elías; Novoa Ramírez, Eliana; y Villagómez Páucar, Alberto (2011). Metodología de la investigación cientifica y asesoramiento de tesis. Perú: Centro de Producción Editorial e Imprenta de la Universidad Nacional Mayor de San Marcos.

- Organización Mundial del Comercio (2010). Informe sobre el Comercio Mundial. El comercio de recursos naturales.

- O.N.G. Capital Humano y Social (2010). La trata de personas en el Perú.

- Páez Warton, José (2009). El plan de tesis. Perú.

- Ramos Núñez, Carlos (2007). Cómo hacer una tesis de Derecho y no envejecer en el intento (y cómo sustentar expedientes).Perú: Gaceta Jurídica. 